\title{
28 UBIQUITOUS COMPUTING FOR HEALTH AND MEDICINE
}

\author{
Chris Atkinson \\ Centre for Research in Systems in Information Systems \\ The University of Manchester \\ Manchester, U.K. \\ Bonnie Kaplan \\ Yale Center for Medical Informatics \\ Yale University \\ New Haven, CT U.S.A. \\ Kent Larson \\ School of Architecture and Planning \\ Massachusetts Institute of Technology \\ Cambridge, MA U.S.A. \\ Henrique M. G Martins \\ Judge Institute of Management \\ University of Cambridge \\ Cambridge, U.K. \\ Jay Lundell \\ Proactive Health Group \\ Intel Corporation \\ Hillsboro, OR U.S.A. \\ Martin Harris \\ Cleveland Clinic \\ Cleveland, OH U.S.A.
}

Ubiquitous information environments today generally include smart cell phones, GPSs, RFIDs, and sensors inside buildings. If accepted by users, wirelessly connected mobile ICT devices like handhelds/PDAs, tablet PCs, or laptop/notebook PCs can also be seen to constitute a platform for information systems ubiquity at the work place and 
beyond. The growth of these technologies is creating new business models and opportunities while changing our notions - and our places - of computing, communication, and work. Already the distinctions between home and work place, leisure and work, private and public are breaking down.

With the advent of new technologies, similar changes are occurring in health care delivery and the distinction between home and locus of health care. Ubiquitous computing in health care is paving the way in which these new technologies intersect with the institutional, social, and private spheres, blurring areas of public and private life, health and illness, work routines, professionalization, and organizational boundaries while contributing to changes in personal empowerment and identity. Ubiquitous medical devices make it possible to monitor patients in their work, domiciliary, and institutional settings as well as enabling peripatetic clinicians to access clinical information and services for treatment of the patient outside of clinical organizations. These also have the potential to lead to the reconfiguration of clinical practices and, to a certain extent, empower the patient, particularly elderly and disabled people. Home health care devices, for example, make it easier for them to live within their own home or chosen institutional environments. Ubiquitous computing also could change the relationship between primary and secondary care by enabling primary care providers to take a much more active role in the effective management of morbidity outside clinical environments.

Informatics in health care has, to date, focused on biomedical informatics, patient clinical record systems, telemedicine, order entry and results reporting, and health billing and administrative systems. These have been accessed primarily through static devices such as workstations, mainly located in hospitals, clinics, or doctors' offices. The advent of ubiquitous computing has opened up a new domain for health and medical informatics. In the process, old issues take on new aspects, and new issues arise that IS researchers need to appreciate - and learn how to address.

The panel will explore the nature of ubiquitous computing and its capacity to change care both at home and in geographically dispersed organizational settings. Panelists are drawn from academia, industry, and health care, with backgrounds in IS, architecture, medical informatics, and cognitive psychology. They will discuss the nature of ubiquitous computing in health and social care by examining ubiquitous computing in the home, the general practitioner's office, and the hospital in different countries. They also will describe a range of research approaches, from field work, to observation and modeling peripatetic care delivery, to experimental living spaces with embedded sensors. While focusing on health care, the panel will explore such general issues as

- How are ubiquitous computing devices used within domiciliary, work, and dispersed institutional settings?

- What might be the relationship between ubiquitous computing and professional roles; personal identities; community relationships; home, locus of health care, and work; and empowerment of individuals employing these new technologies?

- What future organizational models and clinical processes arise out of the availability of ubiquitous computing? 
- What research epistemologies and methodologies are appropriate for the study of ubiquitous information systems?

- What are the appropriate methods and methodologies for the representation, development, and application of ubiquitous technologies and care environments?

- What can we learn about ubiquitous computing in health care that is relevant to other uses of these technologies?

Bonnie Kaplan, Yale Center for Medical Informatics, will moderate the session.

Chris Atkinson, Centre for Research in Systems in Information Systems, School of Informatics Systems, The University of Manchester, will report on current and future research and development that explores the use of ubiquitous and mobile devices in the diagnosis and monitoring of patients within their domicilary setting by primary care providers. The research and development is based on creating clusters of general practices within the UK's National Health Service. These clusters would pool their diagnostic technologies and specialist medical skills together. They would share ubiquitous patient monitoring technologies and access patient notes, teleconsultations, and clinical images through the NHSnet and telecommunications media. These, in turn, would be augmented with therapeutic care to chronically ill individuals using domiciliary ubiquitous devices and patient monitoring technologies.

Kent Larson, Director, Changing Places, Principle Research Scientist, School of Architecture and Planning, Massachusetts Institute of Technology, will discuss PlaceLab, a highly instrumented apartment-scale shared research facility where new technologies and design concepts can be tested and evaluated in the context of everyday living. The rich sensing infrastructure of the PlaceLab will be used to develop techniques to recognize patterns of sleep, eating, socializing, recreation, etc. Particularly for the elderly, changes in baseline activities of daily living are believed to be important early indicators of emerging health problems, often preceding indications from biometric monitoring. The unique data sets generated by the PlaceLab will aid in the development of activity pattern recognition tools. Techniques will be tested that support the effective human-computer interaction required for the proactive encouragement of healthy behaviors related to diet, exercise, medication adherence, etc.

Henrique M. G. Martins, Judge Institute of Management, University of Cambridge, will discuss hospital organizations, where ubiquitous computing is increasingly manifesting itself via initiatives to implement and deploy mobile information communication technology (MICT) devices like PDAs, tablet PCs, and mounted PCs on carts, with and without wireless support. The assumption is that such devices can enhance clinical work practices. Such, however, deserves to be analyzed, as well as the attitudes and practices of hospital organizations toward the usage of such devices by clinicians. Based on fieldwork data from the United States, United Kingdom, Portugal and Singapore, effects of mobile ICT's on clinical practice by individual doctors will be discussed. At the organizational level, a preliminary categorization of different strategies will be advanced together with illustrative examples from researched cases.

Jay Lundell, Senior Researcher, Proactive Health Group, Intel, will discuss Intel's Proactive Health Group, which explores the ways in which ubiquitous computing can support the daily health and wellness needs of people in their homes and everyday lives. 
They use ethnography to discover the needs and challenges of older adults, particularly those struggling with cognitive decline. Jay will present some of Intel's pervasive sensing technologies and work deploying sensor networks to detect, track, and facilitate the social health of elders.

Martin Harris is the chief information officer and chairman of the Information Technology Division of the Cleveland Clinic Foundation in Cleveland, Ohio, a private, non-profit group practice that includes an academic medical center, outpatient services, a research institute, and an education foundation. He is also the executive director of e-Cleveland Clinic, a series of e-health clinical programs offered over the Internet. 